ISSN 0103-5150

Fisioter. Mov., Curitiba, v. 24, n. 4, p. 697-704, out./dez. 2011 Licenciado sob uma Licença Creative Commons

\title{
Avaliação do desempenho físico e funcional respiratório em obesos
}

\author{
Evaluation of physical performance and \\ functional breathing in obese people
}

\author{
Kadma Karênina Damasceno Soares ${ }^{[\mathrm{a}]}$, Évelim Leal Freitas Dantas Gomes ${ }^{[\mathrm{b}]}$, Alceu Beani Júnior ${ }^{[\mathrm{c}]}$, \\ Luis Vicente Franco de Oliveira $^{[\mathrm{d}]}$, Luciana Maria Malosá Sampaio ${ }^{[\mathrm{e}]}$, Dirceu Costa ${ }^{[\mathrm{f}]}$ \\ [a] Mestranda em Ciência da Reabilitação pela Universidade Nove de Julho (Uninove), São Paulo, SP - Brasil, e-mail: \\ belakarenina@hotmail.com \\ [b] Mestranda em Ciência da Reabilitação pela Uninove, São Paulo, SP - Brasil, e-mail: eveliml@yahoo.com.br \\ [c] Médico especialista em Cirurgia Geral, diretor do Centro Especializado no Tratamento da Obesidade, diretor do Serviço \\ de Cirurgia Geral do Instituto de Assistência Médica ao Servidor Público Estadual (Iamspe)/Hospital do Servidor Público \\ Estadual, São Paulo, SP - Brasil, e-mail: alceubeani@terra.com.br \\ [d] Doutor em Ciências da Saúde pela Universidade de Brasília (UnB), professor do Mestrado em Ciências da Reabilitação da \\ Uninove, São Paulo, SP - Brasil, e-mail: oliveira.lvf@uninove.br \\ [e] Doutorado em Ciências Fisiológicas pela Universidade Federal de São Carlos (UFSCar), professor do Mestrado em \\ Ciências da Reabilitação da Uninove, São Paulo, SP - Brasil, e-mail: lucianamalosa@uninove.br \\ [f] Doutor em Ciências Biológicas pela Universidade Estadual de São Paulo (Unesp), professor do Mestrado em Ciências da \\ Reabilitação da Uninove, São Paulo, SP - Brasil, e-mail: dcosta@uninove.br
}

\section{Resumo}

Introdução: 0 desempenho físico tem se tornado importante objeto de estudo em obesos para a avaliação pré-operatória de cirurgia bariátrica. Contudo, ainda há escassez de estudos, especialmente, no que se refere aos testes de campo ou de capacidade funcional. Objetivo: comparar os resultados do Teste de Caminhada de seis Minutos (TC6') com os do Shuttle Walk Test (SWT) em mulheres obesas, juntamente com as respostas do questionário HAQ-20. Metodologia: avaliaram-se 13 mulheres, com idade média de $44 \pm 11$ anos e com Índice de Massa Corpórea (IMC) $43 \pm 5 \mathrm{~kg} / \mathrm{m}^{2}$, submetidas, aleatoriamente e de forma consecutiva, a TC6', SWT, espirometria, força muscular respiratória e questionário HAQ-20. Resultados: Constatou-se que as distâncias apresentaram-se significativamente diferentes (TC6' = $489 \pm 129$ m e SWT $=244 \pm 161 \mathrm{~m}$ ), sendo que o score do HAQ-20 apresentou uma mediana de 1,125. Quando verificada 
a correlação do HAQ-20 com TC6' e o SWT, esta foi constatada apenas para o domínio vestir-se com os resultados do TC6. Conclusão: Com base nesses resultados, conclui-se que a distância percorrida no TC6' foi maior que no SWT, o que sugere melhor aplicabilidade do TC6' em obesos, sendo, nesse caso, possivelmente mais sensível na avaliação da capacidade funcional que o SWT. Os resultados do HAQ-20 foram pouco discriminadores, pois um domínio entre oito mostrou-se significativo quando correlacionado com as distâncias percorridas.

Palavras-chave: Obesidade. Teste de esforço. Caminhada. Qualidade de vida. Espirometria.

\section{Abstract}

Introduction: The physical performance has become an important object of study in obese people for the preoperative evaluation of bariatric surgery. However, there is still lack of studies, especially those regarding field tests or functional capacity. Objective: to compare the results of the Six Minute Walk Test (6MWT) and the Shuttle Walk Test (SWT) in obese women, along with the answers in Health Assessment Questionnaire (HAQ-20). Methodology: Thirteen women with mean age of $44 \pm 11$ years and mean body mass index (BMI) of $43 \pm 5 \mathrm{~kg} / \mathrm{m}^{2}$ were evaluated and submitted, randomly and consecutively, to the $6 M W T$, SWT, spirometry, strength respiratory muscle and HAQ-20 questionnaire. Results: We found that the distances were significantly different ( $6 M W T=489 \pm 129 \mathrm{~m}$ and $\pm 161 \mathrm{~m}$ SWT $=244$ ), and the score of the HAQ-20 showed a median of 1.125. When verified the correlation of the HAQ-20 with $6 M W$ and the SWT, this was only observed for the field dressing with the 6MWT. Conclusion: Based on these results it is concluded that the distance covered in 6MWT was higher than in SWT, which suggests a better applicability of the 6MWT in obese and in this case, possibly more sensitive in the assessment of functional capacity than the SWT. The results of the HAQ-20 were less discriminating as one field of eight was significant when correlated with the walked distances.

Keywords: Obesity. Exercise testing. Walking. Quality of life. Spirometry.

\section{Introdução}

A obesidade caracteriza-se pelo aumento do Índice de Massa Corpórea (IMC), sobretudo pelo excesso de gordura corporal (1), sendo a doença metabólica mais antiga que se conhece. Ela se enquadra no grupo de doença crônico-degenerativa não transmissível (DCNT) e não infecciosa, sendo um dos mais graves problemas de saúde pública, especialmente em países em desenvolvimento, o que levou à condição de epidemia global (2).

Acredita-se que o fato de estar intrinsecamente relacionada ao aumento do IMC influencie negativamente na capacidade física e na função respiratória, independentemente da idade. Isso se deve à maior quantidade de adipocinas produzidas pela gordura visceral que eleva a resistência periférica da insulina, levando ao aumento da pressão arterial $(3,4)$. Além desse fato, é possível observar alterações respiratórias crônicas como: hipoventilação, redução da capacidade aeróbica, da força e endurance dos músculos respiratórios. Essas alterações são causadas pelo acúmulo de tecido adiposo no tórax e cavidade abdominal, fazendo com que ocorra compressão do tórax, do diafragma e do pulmão, o que, por consequência, ocasiona a redução dos volumes pulmonares e acarreta alterações musculares $(5,6)$.

Também é por causa do acúmulo de gordura torácica e abdominal que ocorre a redução na mobilidade da parede do tórax e do músculo diafragma, fazendo com que a complacência torácica seja reduzida e o trabalho respiratório aumentado, causando redução do volume pulmonar e o aumento do recuo elástico do tecido pulmonar (7-9). Tem sido sugerido, também, que a redução da Ventilação Voluntária Máxima (VVM) favorece o aumento das áreas de atelectasia, prejudicando a relação ventilação-perfusão, acarretando hipoxemia arterial e alteração da musculatura respiratória em obesos $(7,10-13)$.

As alterações respiratórias e funcionais podem também ser observadas durante testes de aptidão física, de campo, como o Teste de Caminhada de Seis Minutos (TC6') e o Shuttle Walk Test (SWT). Esses testes têm sido empregados em diversas populações 
para se detectarem possíveis alterações da aptidão física (13). 0 TC6' é um teste submáximo, de simples aplicação, de baixo custo e bem tolerado por pacientes portadores de doenças cardiorrespiratórias, inclusive de obesidade (14). Já o SWT é um teste incremental, portanto máximo e, como tal, empregado com certas limitações. Ambos os testes podem ser empregados para mensurar a capacidade física e funcional em diversas situações de disfunção física inclusive em obesos (15). Contudo, não há evidências sobre sua aplicação nesse tipo de população.

Além dos danos já mencionados ocasionados à função cardiorrespiratória, a obesidade associa-se à redução do bem-estar psíquico, dificultando a interação social e favorecendo a baixa autoestima, o que é determinante na estigmatização do obeso (3). Essas alterações podem ser detectáveis com a aplicação de questionários de qualidade de vida ou da capacidade funcional. 0 Health Assessment Questionnaire (HAQ20) avalia a capacidade funcional, originalmente para portadores de artrite reumatoide (16), a partir de 20 perguntas, distribuídas em oito domínios, também aplicáveis na população de obesos (5).

Tendo em vista a carência de referências sobre esses testes nos obesos, bem como a necessidade de se verificar a função física e funcional desses sujeitos, justificou-se a realização deste estudo, especialmente com o objetivo de comparar os resultados do SWT com os do TC6', explorando também a espirometria, a força muscular respiratória e as respostas do HAQ-20.

\section{Materiais e métodos}

Este estudo foi caracterizado como do tipo transversal. Partiu de uma amostra inicial de 17 obesos, dos quais 4 foram excluídos, sendo 1 por não concluir o SWT, 2 por serem do sexo masculino e 1 por não ter realizado o TC6'. Dessa forma, a amostra final foi composta de 13 mulheres obesas de grau II ou acima deste, com indicação de cirurgia bariátrica e idade entre 18 e 65 anos, aptas a realizarem os testes físicos e funcionais respiratórios, que assinaram o termo de consentimento livre e esclarecido. Com isso, foram estabelecidos os seguintes critérios de exclusão: IMC menor que $35 \mathrm{~kg} / \mathrm{m}^{2}$, alterações cognitivas e físicas que impedissem a realização dos testes, portadores de comorbidades do sistema respiratório, usuárias de drogas ou álcool, que já tivessem sido submetidas anteriormente à cirurgia de tórax e que não concordaram em assinar do termo de consentimento livre e esclarecido.

Após aprovado pelo comitê de ética em pesquisa da instituição sob o n. 336519 e após a seleção das obesas eleitas para cirurgia bariátrica, em um hospital do público do estado de São Paulo, elas foram esclarecidas quanto aos procedimentos e objetivos deste estudo e assinaram o termo de consentimento livre e esclarecido antes de serem submetidas às avaliações.

Após serem avaliadas pela espirometria, por meio das manobras de Capacidade Vital Lenta (CVL), Capacidade Vital Forçada (CVF) e Ventilação Voluntária Máxima (VVM), com um espirômetro portátil da marca Easy One ${ }^{\circledR}$ (Modelo2001) e pela manovacuometria (força muscular respiratória) por um manovacuômetro analógico da marca Wika ${ }^{\circledR}$, as obesas realizaram o TC6' com base no guideline da American Thoracic Society (ATS)(14) e, em seguida, com intervalo mínimo de 30 minutos realizaram o SWT, no qual caminharam entre cones, com uma distância de 10 metros entre eles, em ritmos crescentes, em doze níveis, a partir de um sinal sonoro (15). Em todos os testes físicos, no início e no final, foram mensuradas a pressão arterial (PA), frequência cardíaca (FC), frequência respiratória (f), saturação periférica de oxigênio $\left(\mathrm{SpO}_{2}\right)$, sensação de dispneia e cansaço de membros inferiores, pela escala de Borg.

Em seguida foi aplicado um questionário de capacidade funcional (HAQ-20) (16), composto de 20 questões diretas, distribuídas em 8 domínios (vestirse, levantar-se, alimentar-se, caminhar, higiene pessoal, alcançar objetos, apreender objetos e outras atividades), no qual a maior pontuação é obtida a partir da mediana dos domínios, variando de 0 a 3 pontos. Nesse questionário, menor pontuação reflete a melhor capacidade funcional.

Os dados coletados foram dispostos em tabelas, por média e desvio padrão, e submetidos ao teste de Komolgorov Smirnov (KS) para a verificação de sua distribuição. Para comparar os resultados do TC6' com os do SWT, foi aplicado o teste-t não pareado. Para verificar a existência de correlação entre os testes de esforço e o HAQ-20, foi utilizada a correlação de Spearman. Para verificar a correlação entre a distância percorrida no TC6'e SWT com a FC, a VVM e o IMC, utilizou-se a correlação de Pearson. Para verificar a correlação entre IMC e as pressões respiratórias, foi utilizado a correlação de Pearson. 
E para comparar as pressões respiratórias (PImáx e PEmáx) previstas e obtidas, foi utilizado o teste Anova. Para todos os testes, adotou-se nível de significância de $\mathrm{p} \leq 0,05$.

\section{Resultados}

Os resultados obtidos das 13 mulheres obesas, com idade entre 24 e 62 anos e IMC 36 a $52 \mathrm{~kg} / \mathrm{m}^{2}$, estão apresentados nas Tabelas de 1 a 6, iniciandose pela apresentação de suas características físicas e antropométricas, na Tabela 1.

De acordo com os dados da Tabela 1, pode-se constatar que a amostra foi composta de obesas de grau II e acima deste, com IMC maior ou igual $36 \mathrm{~kg} / \mathrm{m}^{2}$. Lembrando que com um IMC entre 35 e $39,9 \mathrm{~kg} / \mathrm{m}^{2}$ o sujeito é considerado obeso de grau II, e igual ou acima de $40 \mathrm{~kg} / \mathrm{m}^{2}$, obeso de grau III.

Na Tabela 2 estão os valores espirométricos em litros e em porcentagem do predito.

Tabela 1 - Característica físicas e antropométricas

\begin{tabular}{lc}
\hline Idade (anos) & $44 \pm 11(24-62)$ \\
Altura $(\mathrm{cm})$ & $161 \pm 0,06(151-173)$ \\
Peso $(\mathrm{kg})$ & $112 \pm 16(91-139)$ \\
IMC $\left(\mathrm{kg} / \mathrm{m}^{2}\right)$ & $43 \pm 5(36-52)$ \\
\hline
\end{tabular}

Fonte: Dados da pesquisa.

Tabela 2 - Média e desvio-padrão dos valores espirométricos obtidos em litros e em porcentagem do predito

\begin{tabular}{lc}
\hline CVF (L) & $2,83 \pm 0,83$ \\
CVF(\%) & $88 \pm 14$ \\
$\operatorname{VEF}_{1}(L)$ & $2,60 \pm 0,45$ \\
$\operatorname{VEF}_{1}(\%)$ & $91 \pm 14$ \\
CVF/VEF $_{1}(L)$ & $0,84 \pm 0,06$ \\
CVF/VEF $_{1}(\%)$ & $103 \pm 6$ \\
VVM (L) & $92 \pm 14$ \\
VVM (\%) & $70 \pm 13$ \\
\hline
\end{tabular}

Legenda: CVF = Capacidade Vital Forçada; CVF (\%) = Porcentagem da Capacidade Vital Forçada; $\mathrm{VEF}_{1}=$ Volume Expiratório Final no 1 segundo; $\mathrm{VEF}_{1}(\%)=$ Porcentagem do Volume Expiratório Final no primeiro segundo; VVM = Ventilação Voluntária Máxima; VVM (\%) = Porcentagem da Ventilação Voluntária Máxima.

Fonte: Dados da pesquisa.
Como pode ser observado pelos resultados da Tabela 2, não foram constatadas obstrução ou restrição pulmonar, de forma que os obesos não apresentaram alterações de fluxos ou volumes pulmonares e, como tal, podem ser considerados sem alterações pulmonares e de vias aéreas.

A seguir foram comparadas as distâncias percorridas entre os testes de campo (SWT e TC6'), conforme os dados da Tabela 3.

Como pode ser observado nos resultados da Tabela 3, os obesos caminharam significativamente mais no TC6'.

Considerando-se a existência de uma equação que prediz a distância percorrida no TC6'(18) e no SWT (19), comparou-se a distância obtida no TC6' e SWT com a distância predita $(18,19)$, como demonstrado na Tabela 4.

De acordo com os resultados da Tabela 4, constatou-se que não houve diferença significativa entre a distância percorrida no TC6' e a distância predita. Contudo, foi constatada diferença significativa entre a distância percorrida no SWT e a distância predita para esse teste.

Ao se correlacionar a distância percorrida no TC6' com a no SWT, bem como com a distância predita destes com a VVM em litros/minuto e em porcentagem do predito, constatou-se que houve correlação positiva apenas entre as distâncias preditas no TC6' e SWT com a VVM predita. Contudo, entre os valores obtidos e percentuais do previsto não houve.

Tabela 3 - Média e desvio-padrão da distância percorrida no TC6' e no SWT

\begin{tabular}{cccc}
\hline Testes & TC6 $(\mathrm{m})$ & SWT $(\mathrm{m})$ & p \\
\hline DP $(\mathrm{m})$ & $489 \pm 129(366-764)$ & $244 \pm 161(177-554)$ & $0,0004^{*}$ \\
\hline
\end{tabular}

Legenda: DP = distância percorrida.

Fonte: Dados da pesquisa.

Tabela 4 - Média e desvio-padrão da distância percorrida e da prevista $(17,18)$ no TC6' e no SWT

\begin{tabular}{lccc}
\hline & Distância obtida & Distância predita & p \\
\hline TC6 & $489 \pm 129$ & $493 \pm 63$ & ns \\
SWT & $294 \pm 121$ & $440 \pm 71$ & $0,007^{*}$ \\
\hline
\end{tabular}

Fonte: Dados da pesquisa. 
Considerando os resultados da Tabela 4, em que a distância percorrida foi significativamente maior no TC6', e tendo em vista que o SWT durou em média $6 \pm 1 \mathrm{~min}$, todas as variáveis de monitoramento de ambos os testes foram comparadas.

Constatou-se que não houve diferença significativa entre os valores iniciais e os finais de $\mathrm{FC}, \mathrm{SpO}_{2}$, bem como entre as pontuações iniciais e as finais da escala de Borg para dispnéia. Não houve diferença também no MMII entre o TC6' e SWT.

Na Tabela 5 estão apresentados os valores obtidos e previstos $(19,20)$ de PImáx e PEmáx.

Como constatado na Tabela 5, a comparação das pressões respiratórias obtidas com as previstas (20, 21) apresentou uma diferença significativa. Ainda foi possível verificar que as pressões respiratórias obtidas foram sempre inferiores às previstas $(20,21)$.

Não foram constatadas correlações entre IMC e as PImáx e PEmáx tanto dos valores obtidos como dos previstos por ambas as equações $(20,21)$, como também não foram constatadas correlações entre o IMC e as distâncias percorridas nos testes SWT e TC6'.

Verificaram-se correlações entre HAQ-20, distância percorrida no TC6' e no SWT apenas para o domínio "vestir-se" em relação ao TC6', no qual se obteve uma mediana de 2 , apesar de não serem constatadas correlações entre os demais domínios do HAQ-20 e as distâncias percorridas nos testes de campo. Para essa amostra, a mediana entre todos os domínios foi de 1,125 , o que significa uma alteração de capacidade funcional moderada.

\section{Discussão}

Com a submissão aos testes espirométricos, foi possível verificar que as obesas não apresentaram características obstrutivas ou restritivas, avaliadas

Tabela 5 - Média e desvio-padrão dos valores obtidos e previstos $(19,20)$ de PImáx e PEmáx

\begin{tabular}{lrcrc}
\hline & Obtido & $\begin{array}{c}\text { Previsto } \\
\text { (Neder) }\end{array}$ & $\begin{array}{r}\text { Previsto } \\
\text { (Costa) }\end{array}$ & $\mathbf{p} \leq$ \\
\hline PImáx & $-87 \pm 22$ & $-132 \pm 5,2$ & $-94 \pm 5$ & $0,0001^{*}$ \\
PEmáx & $98 \pm 28$ & $143 \pm 7$ & $149 \pm 7$ & $0,0001^{*}$ \\
\hline
\end{tabular}

Legenda: ${ }^{*} p \leq 0,05$.

Fonte: Dados da pesquisa. pela $\mathrm{CVF}$ e $\mathrm{VEF}_{1}$, uma vez que os volumes, capacidade e fluxos pulmonares estão dentro da normalidade quando comparados com os valores de sujeitos saudáveis. Assim, as obesas não apresentaram disfunção pulmonar de fluxo, volumes e/ou capacidades pulmonares, como tem sido sustentado $(5,6)$. Ainda assim, os resultados da VVM apresentam-se diminuídos, em concordância com os diversos estu$\operatorname{dos}(7,10-13,15)$ que envolveram espirometria em obesos. Esses resultados foram utilizados predominantemente para comparação com os dos testes de campo, uma vez que a VVM estima a endurance músculo-respiratória.

Tomando-se como base os achados da literatura (10-13) de que a VVM em obesos pode estar diminuída, e o fato de que nossos sujeitos obesos apresentaram valores médios de $70 \%$ da VVM prevista, considera-se a hipótese de os testes de campo serem influenciados por algumas alterações respiratórias. Contudo, ao se correlacionar a VVM com a distância percorrida no TC6' e SWT, não foram encontradas tais correlações entre os respectivos valores observados, exceto quando comparados os valores preditos, da distância percorrida, com os da VVM, de acordo com Neder (18). Isto é, foram constatadas correlações positivas para a distância predita do TC6' e do SWT, e a VVM predita para os obesos estudados. Esses resultados, de certa forma, condizem com alguns achados (6) com os quais os autores afirmam que os valores espirométricos detectam alterações de função respiratória em sujeitos obesos.

Como não encontramos alterações dos pulmões e da caixa torácica pelos volumes pulmonares passivos, nem das vias aéreas pelos fluxos, podemos considerar que os testes de desempenho físico ainda podem fornecer maiores contribuições para avaliação dos sujeitos obesos.

Na literatura sobre esse tema, tem-se considerado que a obesidade influencia negativamente na capacidade física e na função respiratória, independente da idade $(4,5)$. Uma vez que testes de desempenho físico já vêm sendo empregados para verificar e acompanhar o desempenho de sujeitos saudáveis, bem como em portadores de Doença Pulmonar Obstrutiva Crônica (DPOC) (21-23), reforça-se a hipótese de que esses testes de campo podem revelar importantes contribuições para melhor se compreender o desempenho físico dos obesos.

Nossos resultados revelaram também que os sujeitos obesos conseguiram obter maior desempenho 
no TC6' que no SWT demonstrado pela distância percorrida em metros, pois, enquanto caminharam $244 \mathrm{~m}$, em média no SWT, caminharam praticamente o dobro (489 m) no TC6'. Apesar de o resultado de ambos os testes indicarem o desempenho físico, é sabido que SWT, por ser um teste incremental, assemelha-se ao teste de esforço; portanto, fornece mais informações da condição aeróbia que o TC6'(24), sendo que este último, por respeitar mais o ritmo do sujeito avaliado, parece melhor se adaptar para essa população, que nem sempre consegue impor velocidade ou alteração da velocidade em seu deslocamento. Esse aspecto é justificado pelo aumento do IMC e, consequente, dificuldade de sustentação do próprio peso corporal. Esses resultados contribuem para a informação de que o TC6' pode ser mais apropriado para os obesos.

Quando comparadas as distâncias percorridas com a prevista (18) no TC6', não constatamos diferença significativa. Esse resultado se assemelha aos achados de Soares et al. (25), nos quais a distância média percorrida para mulheres foi de $495,19 \pm 54,01$, próxima da de nosso achado, que foi de $489 \pm 129 \mathrm{~m}$. Já em relação ao SWT, constatamos diferença entre a distância obtida e a predita (19), corroborando com os achados de Jurgensen et al. (19), os quais relataram que, quanto maior o IMC, menor a distância percorrida no teste SWT. Esses resultados reforçam a hipótese de que, por tratar-se de um teste incremental, o SWT não é o mais adequado para os sujeitos obesos.

Portanto, com base nesses resultados, pode-se considerar que, ao se estabelecer que a distância percorrida pode determinar o desempenho físico do obeso e que o TC6' se apresenta mais adequado para avaliar o seu desempenho físico, uma vez que se trata de um teste submáximo, este não requer carga de trabalho extrema e é melhor tolerado que SWT.

Esses argumentos se sustentam pelos dados observados no monitoramento do TC6' e do SWT, sobretudo pela média de tempo no SWT, que foi de 6 \pm 1 minutos, semelhante ao tempo estabelecido no TC6', que é de seis minutos. Analisando as correlações da distância percorrida nos testes de campo, com as variáveis mensuradas nestes $\left(\mathrm{FC}, \mathrm{SpO}_{2}\right.$, Borg dispneia e MMII), foi possível constatar uma correlação positiva para a FC inicial e final de ambos, FC inicial do TC6' e Borg dispneia final no SWT. Apesar desses resultados fisiológicos, detectaram-se maiores alterações quanto à escala de Borg para dispneia no SWT. Isso indica certa homogeneidade nos esforços realizados em ambos os testes, reforçando a maior aplicabilidade do TC6' nos obesos.

Outro dado interessante foi a falta de correlação entre a distância percorrida nos testes de campo com o IMC e a $\mathrm{SpO}_{2}$, diferentemente dos resultados em estudos realizados com sujeitos saudáveis, com idade entre 50-85 anos (26), em que os autores encontraram uma correlação negativa, ou seja, quanto maior o IMC, menor era a distância percorrida no TC6', o que também foi confirmado por Jurgensen et al. (19) entre o IMC e a distância percorrida no SWT. Esses autores concluíram que a carga de trabalho é influenciada pelo aumento do peso e IMC, ou seja, quanto maior o IMC, menor será a distância percorrida no SWT. Não discordamos dessa fundamentação, contudo, não constatamos tais correlações ao comparar os testes de nossa amostra.

Da mesma forma, não encontramos correlação entre o IMC e a força muscular respiratória, medidas pelas PImáx e PEmáx, o que está de acordo com os resultados observados em 51 obesos quanto a função pulmonar, força muscular respiratória e endurance (27). Apesar desses resultados, constatamos diferença significativa entre a PImáx e PEmáx obtidas e previstas $(17,18)$, sendo os valores observados inferiores aos previstos, o que está de acordo com os achados de Weiner et al. (28) em obesos também antes da cirurgia bariátrica.

Em relação à capacidade funcional "subjetiva", esta foi avaliada por meio de um questionário de capacidade funcional (HAQ-20) (16), composto de 20 questões diretas, distribuídas em 8 domínios (vestirse, levantar-se, alimentar-se, caminhar, higiene pessoal, alcançar objetos, apreender objetos e outras atividades). Sua pontuação é obtida por meio do cálculo da mediana dos domínios do questionário, variando de zero a três, sendo que, quanto menor a pontuação, melhor a capacidade funcional; e quanto maior sua pontuação, pior a capacidade funcional demonstrada $(29,30)$.

Assim, é estabelecido que um HAQ elevado é um mau fator prognóstico para abandono de trabalho, em que a média aritmética de todos os domínios pode variar de $0-1$, demonstrando uma alteração na capacidade funcional leve, 1-2 moderada e 2-3 grave (30). Nossos resultados revelaram uma mediana de 1,125 , que caracteriza um distúrbio de capacidade funcional moderado. A capacidade funcional, mensurada pelo HAQ-20, é uma variável ampla que poderá estar relacionada com a forma como a pessoa 
percebe o desempenho de suas atividades de vida diária (30) e também com o acúmulo de gordura corporal (12). Como tal, deve ser considerada como uma importante ferramenta de avaliação coadjuvante aos testes físicos de campo, especialmente nos obesos, que normalmente apresentam certo mal-estar psíquico pela própria obesidade.

\section{Conclusão}

Com base nesses resultados, em especial pelo fato de a distância percorrida no TC6' ter sido acentuadamente maior no SWT, e nas alterações fisiológicas identificadas, pode-se concluir que o primeiro refletiu melhor as condições físicas e funcionais das obesas e, portanto, melhor se aplica nesta avaliação. A diminuição da função pulmonar em obesos, embora amplamente comentada na literatura, não parece ser caracterizada como tal, exceto ao se considerar uma única variável da espirometria, a VVM. De forma semelhante, o IMC não se correlacionou com os resultados da distância percorrida nos testes de campo.

A força muscular respiratória, medida pelas PImáx e PEmáx, apresentou-se reduzida nos obesos em comparação com valores de referência para sujeitos saudáveis, não obesos. Todavia, não se correlacionou com o IMC.

A obesidade causa certos desconfortos no desempenho físico que nem sempre são detectados por meio de testes que avaliam variáveis fisiológicas ou mecânicas. Quando estes são manifestados por meio de respostas padronizadas em ferramentas específicas, como o HAQ-20, utilizado neste estudo, embora seja detectado esse desconforto, apenas em alguns domínios, como foi o caso, podem fornecer importantes informações, nem sempre mensuradas por variáveis objetivas.

Esse aspecto requer novos estudos, especialmente porque os diferentes domínios dos questionários em geral, e em especial do HAQ-20, podem também estar relacionados às atividades de vida diária desses sujeitos, não avaliados neste estudo.

\section{Agradecimentos}

À equipe do Laboratório de Avaliação Funcional Respiratória e ao CNPq Proc n. 478147/08-2, pela viabilidade dos equipamentos.
À equipe médica do Hospital do Servidor Público Estadual de São Paulo (HSPE).

\section{Referências}

1. Gregg EW, Cheng YJ, Cadw El BL, Imperatore G, Willians DE, Flegal KM. Secular trends in cardiovascular disease risk factors according to body mass index in us adults. JAMA. 2005;293(15):1868-74.

2. Porto EBS, Morais TW, Raso V. Avaliação do nível de conhecimento multidisciplinar dos futuros profissionais na propedêutica da obesidade. Rev Bras Nutr Emagrecimento. 2007;2(1):67-71.

3. Orsi JVA, Nahas FX, Gomes HC, Andrade CHV, Veiga DF, Novo NF, et al. Impacto da obesidade na capacidade funcional de mulheres. Rev Assoc Med Bras. 2008;54(2):106-9.

4. Faintuch J, Souza SAF, Valezi AC, Sant'Anna AF, Rodrigues JJG. Pulmonary function and aerobic capacity in asymptomatic bariatric candidates with very severe morbid obesity. Rev Hosp Clin Fac Med. 2004;59(4):181-6.

5. Costa D, Barbalho MC, Miguel GPS, Forti EMP, Azevedo JLMC. The impact of obesity on pulmonary function in adult women. Clinics. 2008;63(6):719-24.

6. Ogunnaike BO, Jones SB, Jones DB, Provost D, Whitten CW. Anesthetic considerations for bariatric surgery. Anesth Analg. 2002;95(6):1793-805.

7. Deane $S$, Thomson A. Obesity and the pulmonologist. Arch Dis Child. 2006;91(2):188-91.

8. Jones RL, Nzekwu MMU. The effects of body mass index on lung volums. Chest. 2006;130(3):827-33.

9. Ladosky W, Botelho MAM, Albuquerque JP. Chest mechanics in morbidly obese non-hypoventilation patients. Respir Med. 2001;95(4):281-6.

10. Powers MA. The obesity hipoventilation syndrome. Respir Care. 2008;53(12):1723-30.

11. Casas A, Pavía J, Maldonado D. Respiratory muscle disorders in chest wall diseases. Arch Bronconeumol. 2003;39(8):361-6.

12. Séres L, Ayerbe J, Coll R, Rodríguez O, Manresa JM, Marrugat J, et al. Cardiolpumonary function and exercise capacity in patients with morbid obesity. Rev Esp Cardiol. 2003;56(6):594-600. 
13. Deboeck G, Niset G, Vachiery JL, Moraine JJ, Naeije R. Physiological response to the six-minute walk test in pulmonary arterial hypertension. Eur Respir J. 2005; 26(4):667-72.

14. American Thoracic Society - ATS. Statement: guidelines for the six-minute walk test. Am J Respir Crit Care Med. 2002;166(1):111-7.

15. Rosa FW, Camilier A, Mayer A, Jardim JR. Avaliação da capacidade de exercício em portadores de doença pulmonar obstrutiva crônica: comparação do teste de caminhada com carga progressiva com o teste de caminhada com acompanhamento. J Bras Pneumol. 2006;32(2):106-13.

16. Ferraz MB. Tradução para o português e validação do questionário para avaliar a capacidade funcional "Stanford Health Assessment Questionnarie" [tese]. São Paulo: Universidade Federal de São Paulo; Escola Paulista de Medicina; 1990.

17. Enright PL, Shuerril DL. Reference equations for the six minute walking in healthy adults. Am J Respir Crit Care Med. 1998;158(5 Pt 1):1384-7.

18. Jurgensen SP, Antunes LC, Tanni SE, Banov MC, Lucheta PA, Bucceroni AF, et al. The Incremental shuttle walk test in older Brazilian adults. Respiration. 2011;81(3):223-8.

19. Neder JA, Andreoni S, Lerario MC, Nery LE. Reference values for lung function II. Maximal respiratory pressures and voluntary ventilation. Braz J Med Biol Res. 1999;32(6):719-27.

20. Costa D, Gonçalves HA, de Lima LP, Ike D, Cancelliero KM, Montebelo MI. New reference values for maximal respiratory pressures in the Brazilian population. J Bras Pneumol. 2010;36(3):306-12.

21. Iwama AM, Andrade GN, Shima P, Tanni SE, Godoy I, Dourado VZ. The six minute walk test and body weight walk distance product in Brazilian subjects. Braz J Med Biol Res. 2009;42(11):1080-5.

22. Signg SJJ, Evans PW, Morgan MDL. Minimum clinically important improvement for the incremental shuttle walkig test. Thorax. 2008;63(9):775-7.
23. Souza SA, Faintuch J, Fabris SM, Nampo FK, Luz C, Fabio TL, et al. Six-minute walk test: functional capacity of severely obese before and after bariatric surgery. Surg Obes Relat Dis. 2009;5(5):540-3.

24. Signg SJJ, Evans PW, Morgan MDL. Minimum clinically important improvement for the incremental shuttle walkig test. Thorax. 2008;63(9):775-7.

25. Soares CPS, Pires SR, Brito RR, Parreira VF. Avaliação da aplicabilidade da equação de referência para estimativa de desempenho no teste de caminhada de 6 minutos em indivíduos saudáveis brasileiros. Rev Soc Cardiol do Estado de São Paulo. 2004;1(Supl A):1-8.

26. Troosters T, Grosselink R, Decramer M. Six minute walking distance in healthy elderly subjects. Eur Respir J. 1999;14(2):270-4.

27. Sarikaya S, Cimen OB, Gokcay Y, Erden R. Pulmonary function tests, respiratory muscle strength, and endurance of persons with obesity. Endocrinologist. 2003; 13(2):136-41.

28. Weiner P, Waizman J, Weiner M, Rabnet M, Magadle $\mathrm{R}$, Zamir D. Influence of excessive weight loss after gastroplasty for morbid obesity on respiratory muscle performance. Thorax. 1998;53(1):39-42.

29. Oliveira LM. Evolução da capacidade funcional de pacientes com artrite teumatóide, avaliada pelo "Stanford Health Assesment Questionnaire" e Escala EPM-ROM [dissertação]. São Paulo: Universidade Federal de São Paulo, Escola Paulista de Medicina; 1996.

30. Corbacho MI, Dapueto JJ. Avaliação da capacidade funcional e da qualidade de vida e de pacientes com artrite reumatóide. Rev Bras Reumatol. 2010;50(1): 31-43. doi: 10.1590/S0482-50042010000100004.

Recebido: $16 / 11 / 2010$ Received: $11 / 16 / 2010$

Aprovado: 24/05/2011 Approved: 05/24/2011 